\title{
Distributed knowledge management portal for Learning Organizations with collaborative environment
}

\begin{abstract}
A knowledge management system (KMS) is a concept that can be used for creating knowledge repositories, improving knowledge access, sharing and communicating through collaboration, enhancing the knowledge environment and managing knowledge as an asset for an organization as well as inter-organization especially in Learning Organization (LO). In this paper, we will discuss and propose a distributed KMS portal for LOs with collaborative environment, a model and its components of KMS portal in LOs that will help the organizations to increase its productivity and quality as well as to gain return on investment (ROI). The component of KMS portal consists of its functionality, architecture, taxonomy, psychological, sociocultural and audit.
\end{abstract}

Keyword: Learning Organization (LO); Knowledge management system (KMS) portal; Collaborative environment 